\title{
Simulation \& Performance Analysis of Wired and Wireless Computer Networks
}

\author{
Rahul Malhotra \\ Associate Professor, \\ Adesh Institute of Engineering \& \\ Technology, Faridkot
}

\author{
Vikas Gupta \\ Research Scholar, \\ Adesh Institute of Engineering \& \\ Technology, Faridkot
}

\author{
Dr. R. K. Bansal \\ Associate Professor, \\ Giani Zail Singh College of \\ Engineering \& Technology, Bathinda
}

\begin{abstract}
In this paper, performance analysis of the Wireless and Wired computer networks through simulation has been attempted using OPNET as simulating tool. For wired networks, the performance parameters like delay and throughput have been investigated with varying transmission links and load balancers. The load-balancing has been analyzed through parameters like analysis of traffic sent and traffic received. While in wireless networks the metrics like delay, retransmission attempts and throughput have been estimated with varying physical characteristic and buffer size. From the obtained results, it is gathered that performance of the wired networks is good if high speed Ethernet links like 1000 Base $\mathrm{X}$ and server-load balancing policy are used whereas the performance of Wireless LAN can be improved by fine tuning and properly choosing the WLAN parameters. For the tested simulation scenarios the performance is observed to be better with wireless networks using infra-red type physical characteristics and higher buffer size (1024Kb).
\end{abstract}

\section{Keywords}

OPNET, load-balancing, physical characteristics, buffer size.

\section{INTRODUCTION}

Computer Network is an interconnection of computers for sharing of data and network resources. With the evolution of Internet, the networking technology has not been just confined to resource sharing but has boomed in the arena of cellular and mobile communication as well. Rapid development in the field of very large scale integration of complex circuits on to a smaller chip has led to the evolution of high speed computer networks. The traditional wired transmission medium poses constraints like mobility and extensive cabling. But wireless communication is a flexible data communication system implemented as an extension to or as an alternative for wired communication. The bandwidth and the services provided by the wireless communication networks are similar to that provided by the wired networks. So, as the networks are being upgraded from scratch all over the world, network planning is becoming all the more important. Computing the viability and performance of computer networks in real can be very expensive and painstaking task. To ease and comfort the process of estimating and predicting a network design, simulation and modeling techniques are widely used and put into practice. The network simulation thus becomes an indispensable tool for carrying out the design and redesign operations and for evaluating the performance of the network. A variety of simulation tools like NS-2, NetSim, and OPNET are available for the purpose of modeling and simulation but the choice of a simulator depends upon the features available and the requirements of network application. OPNET is one of the simulation software which can provide statistical analysis of data for network planning and design operations.
In this paper, the wired and wireless networks have been modelled and simulated using OPNET Modeler. The analysis helped to estimate and optimise the performance of wired and wireless networks using the proposed optimization techniques.

\section{RELATED WORK}

An important issue related to the network performance is congestion which may occur in a network when the number of packets sent to the network is greater than the number of packets that the network can handle. The intermediate devices like routers and switches in a network have buffers where the packets wait in a queue before and after processing. Depending on the packet arrival rate and the packet departure rate which may be higher or lesser than the packet processing rate, the size of input or the output queue may increase or decrease. This increase in queue size may lead to congestion. A key issue in designing any good network is to use congestion control mechanism. The congestion control involves two factors that measure the performance of a network: delay and throughput. Efforts have been made to analyse the effect of various parameters on the performance of both wired and wireless networks.

Wired local area networks includes several technologies like Ethernet, token ring, token bus, Fibre distributed data interface and asynchronous transfer mode local area networks. The Ethernet is a contention media access method. In its purest form, contention means that the computers are contending for use of the transmission medium. Any computer in the network can transmit at any time (first come, first serve).

IEEE 802.3 standard specifies CSMA/CD as the access method for first-generation 10-Mbps Ethernet, a protocol that help devices share the bandwidth evenly without having the two devices transmit at the same time on the network medium. When the two devices transmit at the same time the collision can occur. This collision generates a jam signal that causes all nodes on the segment to stop sending data, which informs all the devices that a collision has occurred. The collision also invokes a random back off algorithm (which determines when the colliding stations can retransmit). Each device on the Ethernet segment stops transmitting for a short time until the timers expires. Thus the collisions are overcome. This CSMA/CD protocol was created to overcome the problem of collisions that occur when the packets are transmitted simultaneously from different nodes over the same medium. The CSMA/CD network sustaining heavy collisions causes following effects:

Delay: Back off introduces the transmission delay which is enforced when a collision occurs. The retransmissions are resumed on the expiry of this delay time. 
Low throughput: Throughput in a network is defined as the number of packets passing through the network in a unit of time. The throughput is reduced as a result of collisions.

Congestion: Congestion occurs when load on the network (the number of packets to be sent to the network) is greater than the capacity of the network (the number of packets that a network can handle). The collisions in the network cause the routers and switches to have queues (buffers that hold packets before and after processing). If either packet arrival rate or packet departure rate is higher than the packet processing rate the input/output queue becomes longer, thus leading to congestion. So collisions introduce congestion.

The evolutions of Ethernet to bridged LAN lead to the division of larger LAN into smaller networks and then connecting them by using multi-port Bridges. This provided an advantage of separate collision domains. The evolution from bridged LAN to switched LAN lead to technology of connecting multiple workstations using a device called switch. This causes the bandwidth to be shared between switch and workstation (5 Mbps each) leading to a faster switched Ethernet like 10 Base 2 and 10 Base 5 Ethernets which provide half-duplex communication. But evolution from switched Ethernet (half-duplex) to full-duplex switched Ethernet increased the capacity from 10 to 20 Mbps.

Due to the various drawbacks of wired LANs like extensive cabling and immobility etc., the wireless technology gained momentum. Wireless local area networks (WLAN) enabled people on the move to communicate with anyone, anywhere, at any time, using range of multimedia services. The tremendous growth of cellular telephone and mobile systems coupled with spreading of laptop and palmtop computers indicates a bright future for such networks, both as standalone and as part of large networking infrastructure. The next stage of this development will be complementing or replacing the traditional wired network. Wireless communication technologies employ infrared, spread spectrum and microwave radio transmission techniques with varying data rates. Figure 1 provides an overview of various types of transmission techniques, data rates and network coverage area.

The demand of wireless LAN has increased over a span of time because of its comparative simplicity, flexibility, high rate access and low cost. The wireless network infrastructure is useful to provide accessibility in rough terrains and even rural areas where establishing wired infrastructure is difficult.

Wireless LAN protocol is based on IEEE 802.11 standard. This standard defines a medium access control (MAC) sub layer and three physical (PHY) layers. This protocol describes a wireless LAN that delivers services commonly found in wired networks, e.g. throughput, reliable data delivery and continuous network connections. The architecture of IEEE 802.11 WLAN is designed to support a network where a number of mobile stations are involved. Within the MAC layer, DCF (Distributed Coordination Function) is used as fundamental access method while Point Coordination Function (PCF) is known as Carrier Sense Multiple Access with collision avoidance (CSMA/CA) protocol. It is an asynchronous access method based on the contention for the usage of shared channels.

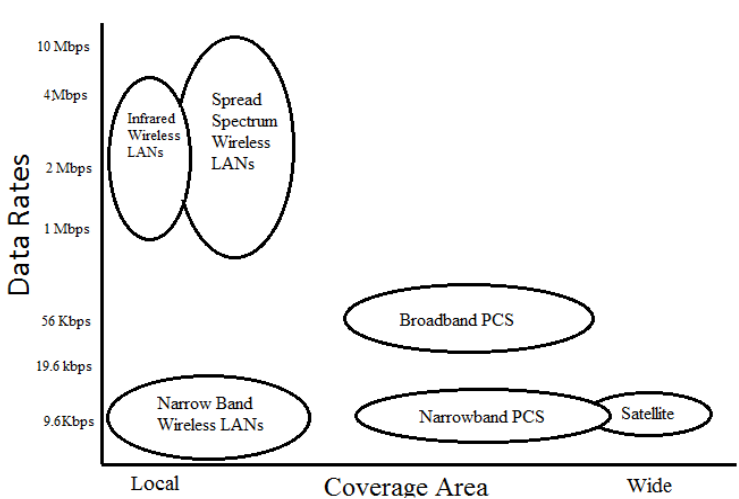

Figure 1 Network coverage vs. coverage area

PCF provides a contention free access mechanism through RTS/CTS (Request to Send/ Clear to Send) exchange. The IEEE 802.11 protocol includes authentication, association and re-association services, an optional encryption/decryption procedure, power management and time-bound transfer of data. Though wireless technology provides convenience and advantages like ease of mobility, scalability and flexibility but it has certain downfalls like:

Speed: The speed of the wireless networks (ranging from 2Mbps to more than $100 \mathrm{Mbps}$ according to the IEEE 802.11n standards) is comparatively less than the wired LANs (available in Gbps). The data rate decreases with the increase in the number of nodes.

Range: The devices operate within a limited distance from an access point. The distance between the devices is determined by the standard used. It is distance between the buildings and other obstacles between the access point and the user.

Cost: The wireless connecting devices are more costly as compared to connecting devices used in wired networks.

Reliability: The wireless networks are subject to interference and can thus pose a problem in the administration of Wireless Infrastructure.

Security: Technically wired LANs are more secure than Wireless LANs. Since wireless signals are transmitted through the air, they can be captured by devices outside the network. However, the majority of wireless local area networks protect their data with the Wired Equivalent Privacy (WEP) encryption standard or Wi-Fi Protected Access (WAP) which makes wireless communications almost as safe as wired ones

Bit Error Rate (BER): The wireless network's media is error prone hence its BER is higher than the wired LANs.

Carrier Sensing: Carrier sensing is difficult in wireless networks because a station is incapable of listening to its own transmissions.

Hidden Terminal Problem: The hidden terminals decrease the performance of the wireless LANs.

Moreover, analysis and optimization is difficult in real but simulation is one of the alternative options for the same. Though wireless networks, in contrary to wired networks, are relatively a new field of research, there exist some simulators to develop networks and test the effect of change in conditions on various performance parameters. This paper has been focused on the estimation of effects on throughput \& delay using varying transmission links, varying physical characteristics, load balancing and buffer size for the wired and wireless networks using OPNET. The proceeding sections involve the implementation of wired and wireless local area network models and the performance analysis of both wired 
and wireless local area networks using OPNET (Optimized Network Engineering Tool).

\section{MODELLING AND SIMULATION}

A model is a logical, physical, mathematical representation of an entity, process, a system or phenomenon. These models are analyzed by the network designers to predict how these networks would perform in real-time. This adoption of low cost simulation techniques helps to overcome expenses and design an accurate network model. Models can be static or dynamic. While static models are not effective for changing environments, the dynamic models are much effective over there.

This dynamic modelling is called simulation. Simulation can be used to model the ideas, evaluate the pros and cons of the network designs, make alternatives and finally choose a better configuration just by sitting at one place i.e. the designers can predict and estimate the performance of the system. It is the replica of a dynamic process within a model to achieve knowledge, which one can carry over to reality. Network simulations allow alternatives to be compared under a wider variety of workloads and environments.

Among the various simulators available, Optimized Network Engineering Tools (OPNET) IT Guru Academic Edition is a simulator which is comprehensive and technology neutral in its capabilities. IT Guru enables the network designers to create a virtual network consisting of relevant hardware, protocols, and application software. This virtual network is a pure software entity that can run on an individual workstation. The network devices like routers, switches etc. can be modelled in IT Guru virtual network. This network can be scaled from a small LAN to wide area network. Once a virtual network has been created it can be manipulated according to the need of the application. The network devices can be changed, removed or inserted into the virtual network as desired to find out the most appropriate configuration and also implement the given application. The effects of various manipulations can be quantifiably examined and analyzed. The OPNET IT Guru provides a GUI to create the virtual network conveniently. OPNET simulator is built on top of discrete event system (DES) and it simulates the system behaviour by modeling each event in the system and processing it through user defined processes. OPNET is very powerful software to simulate heterogeneous network with various protocols. It has several distinct methods of creating topologies. Modeler supports almost all network types and technologies. OPNET runs on top of a $\mathrm{C}$ compiler and provides a GUI. Models are built in hierarchical fashion. OPNET Modeler is based on a series of hierarchical editors that directly parallel the structure of real networks, equipment, and protocols. These editors are Project editor, node editor and process editors.

\section{PERFORMANCE ANALYSIS}

This section describes the performance analysis of wireless and wired computer networks using simulation. The simulation was done using the network simulator - OPNET. In case I: First of all, a comparison was done by varying the types of transmission links (Ethernet links) used in the wired networks for communication between the server and the clients. Secondly, a load balancing mechanism has been used to balance traffic load in the wired network. In this, different load balancing policies were used. Investigations were done to find the policy using which the traffic sent/ received can be balanced to improve the performance. The performance metrics evaluated are delay, throughput, traffic sent and traffic received. In case II: the performance analysis of the wireless computer networks has been illustrated by tuning the Wireless local area network parameters (such as physical characteristics and buffer Size among many other parameters). The performance metrics analyzed are delay and throughput for wireless networks.

\section{Case I: For Wired Networks}

In the simulation scenario shown in Figure 2, comparison has been done by varying the types of transmission links (Ethernet links) used in the networks for communication between the server and the clients in a wired local area network.

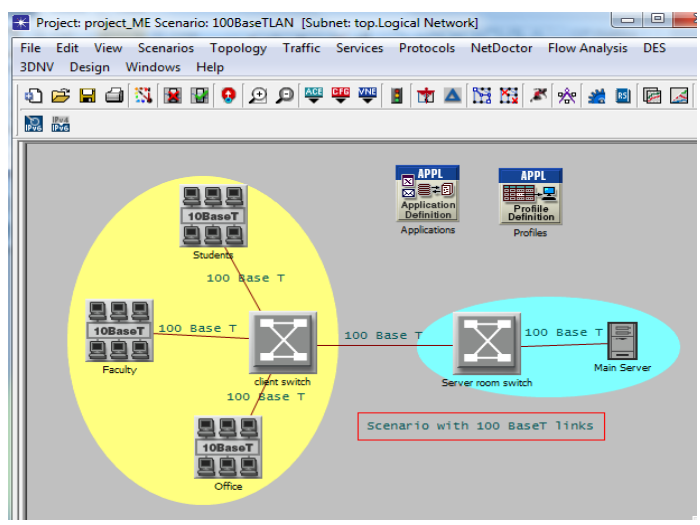

Figure 2 Wired local area network model I

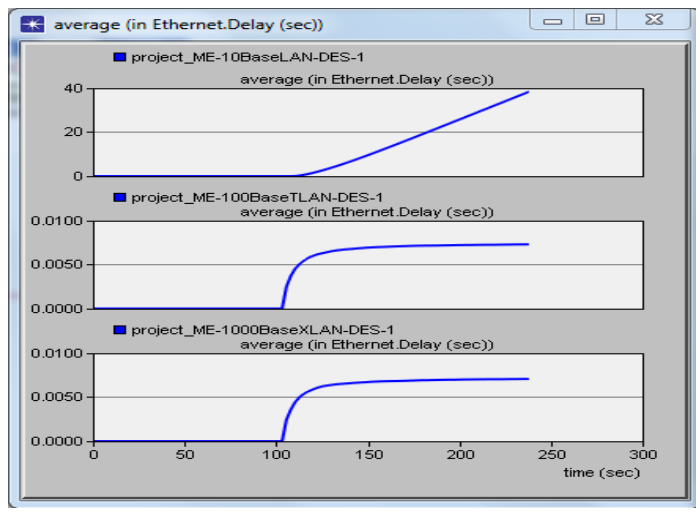

Figure 3 Ethernet Delay (sec)

Figure 2 shows the wired network being modeled and simulated for performance analysis using OPNET. The comparison was made for same number of users but different types of links like 10 Base T, 100 Base T and 1000 Base X. The analysis of performance metrics like Ethernet - Delay shown in figure 3 illustrates that the maximum delay occurs for 10 Base $\mathrm{T}$. The performance analysis in figure 4 of network model shown in figure 2 illustrates the impact of varying types of links on the Ethernet traffic received. The traffic received using 100 Base $\mathrm{T}$ and 1000 base $\mathrm{X}$ is maximum because of the reduction in delay. 


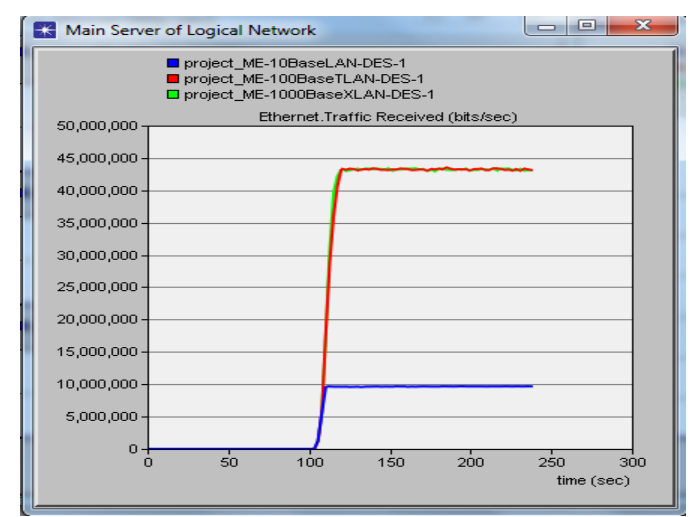

Figure 4 Ethernet traffic received (bits/sec)

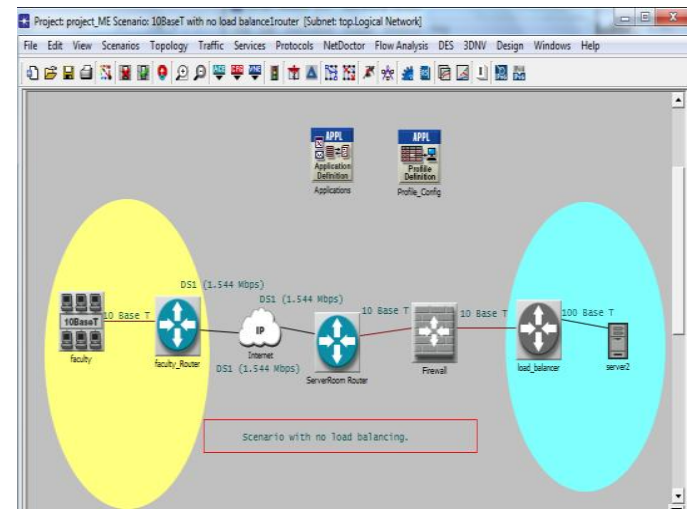

Figure 5 Wired Network using Load Balancer

Now in the simulation scenario shown in Figure 5, the network has been modeled using the load-balancer using various load-balancing policies. When the load balancer receives a packet from a client machine, it must choose the appropriate server to handle the request. The load balancer will use the load balancing policy to determine which server is most appropriate. Following load balancing policies can be used:

Random: The load balancer chooses one of the candidate servers at random.

Round-Robin: The load balancer cycles through the list of candidate servers.

Server Load: The load balancer chooses the candidate server with the lowest CPU load.

Number of Connections: The load balancer keeps track of the number of connections it has assigned to each server. When a new request is made, it chooses the server with the fewest connections. The performance analysis has been done for networks with and without load balancing policy. When no load balancing policy is used, the number of users is varied to vary the network load. Then the performance analysis was done by comparing the networks: one with maximum network load (without load balancing policy) and other network with same maximum network load (with a load balancer implementing random load balancing policy). Figure 6 shows the performance analysis for network with and without load balancer.As can be seen in Figure 6, as the number of user increases, more traffic is generated.

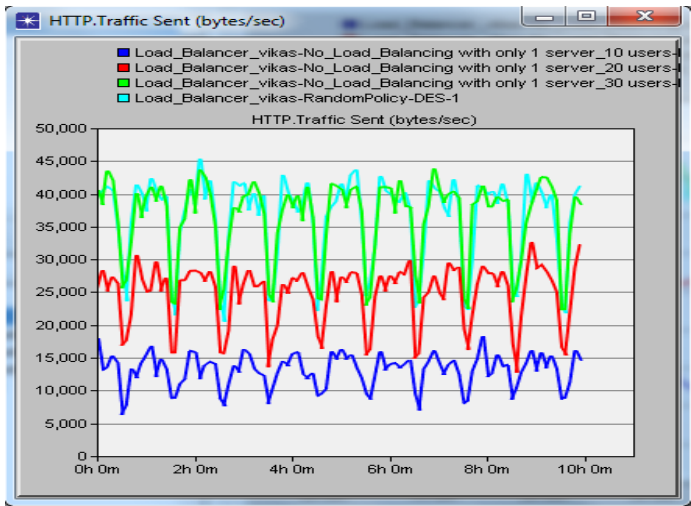

Figure 6 Traffic sent in a wired network

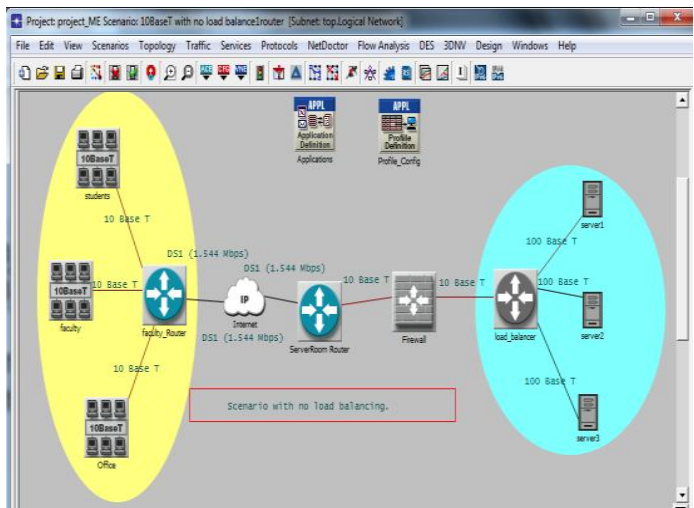

Figure 7 LAN with load-balanced multiple servers

It is also observed that the introduction of load balancing for a single server has no effect on the level of generated traffic. The network model shown in figure 7 shows implementation of wired local area networks using load balanced multiple servers. The performance analysis of the network model shown in figure 7 using various load balancing policies has been illustrated in figure 8 for the traffic sent and Figure 9 for the traffic received.

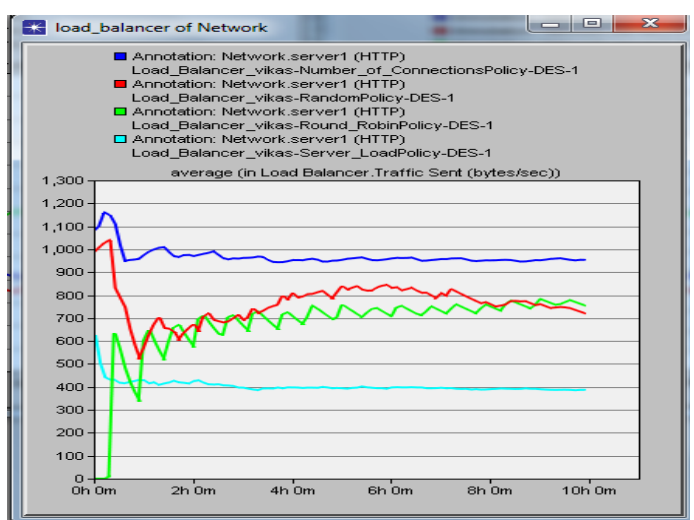

Figure 8 Traffic sent using load balancing policies

The performance analysis is illustrated in figure 8 and figure 9. 


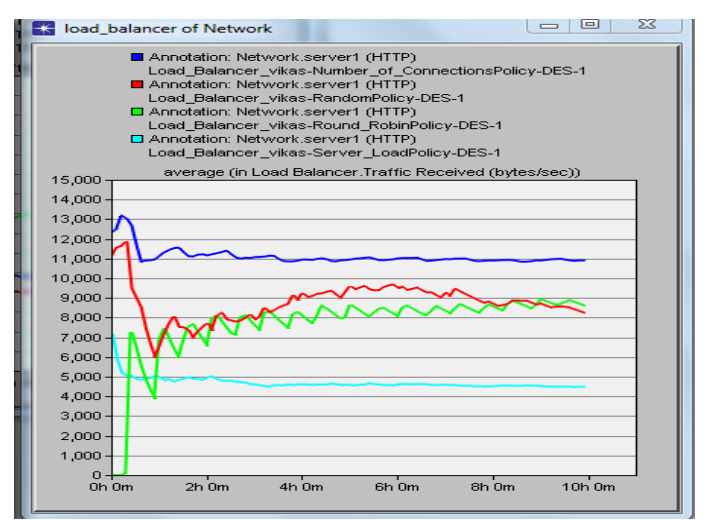

Figure 9 Traffic received using load balancing policies

The investigations present that while average traffic received or sent (in bytes/sec) with the number of connections policy is more than others, the average traffic received or sent (in bytes/sec) with the server-load policy is lesser than others.

\section{Case II: Wireless Local Area Networks}

In this case the wireless local area networks were simulated using OPNET as shown in figure 10.

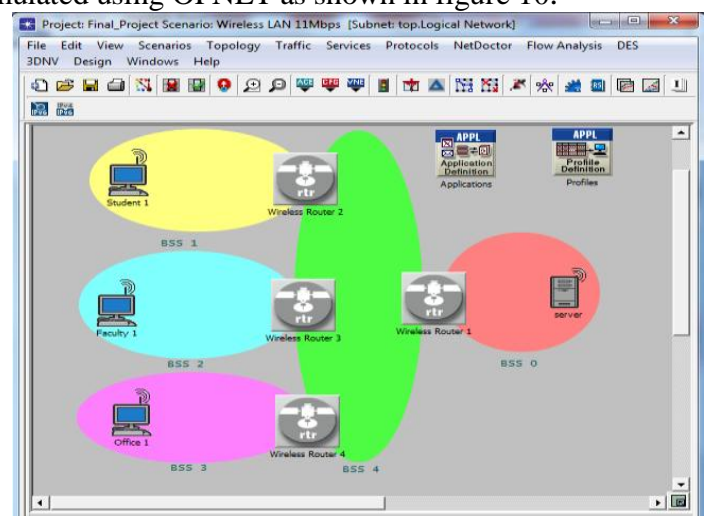

Figure 10 Network Model of the Wireless LAN

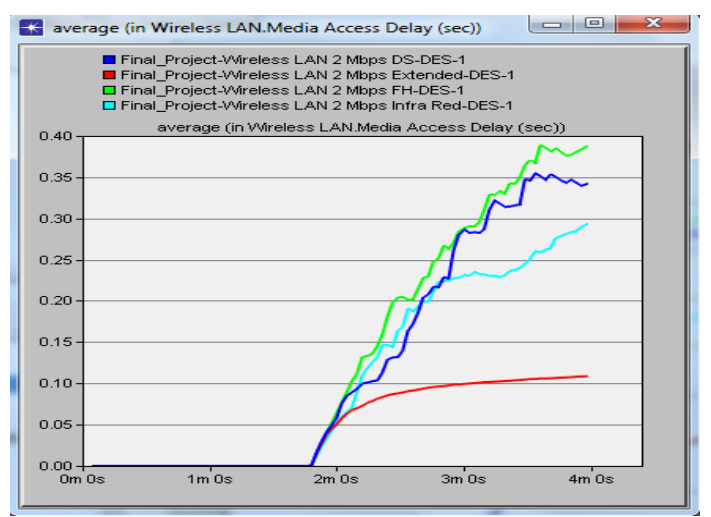

Figure 11 Media Access Delay for varying physical characteristics

The performance analysis of wireless networks can involve the following considerations like RTS Threshold, Fragmentation Threshold, Data rate and buffer size. In this section an insight has been provided on the variations occurring due to the change in physical characteristics and Buffer size. Various scenarios were modelled to estimate the performance of wireless networks at a constant data rate of 2 Mbps but varying physical characteristics like Frequency Hopping, Direct Sequence, Infra red and Extended Rate PHY (IEEE $802.11 \mathrm{~g}$ ). The performance analysis for media access delay has been illustrated in figure 11. The figure 11 shows lower delays for IR and ERP as compared to the higher delays for DSSS and FHSS. For each packet the delay is recorded when the packet is sent to the physical layer for the first time.

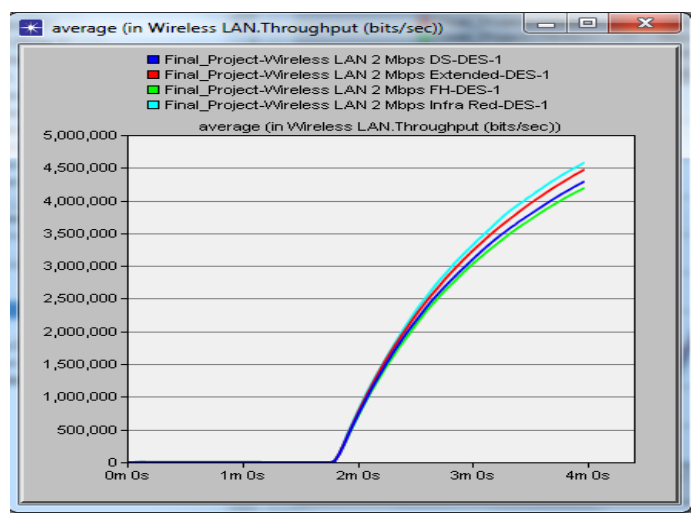

Figure 12 Throughput (bits/sec) for varying physical characteristics

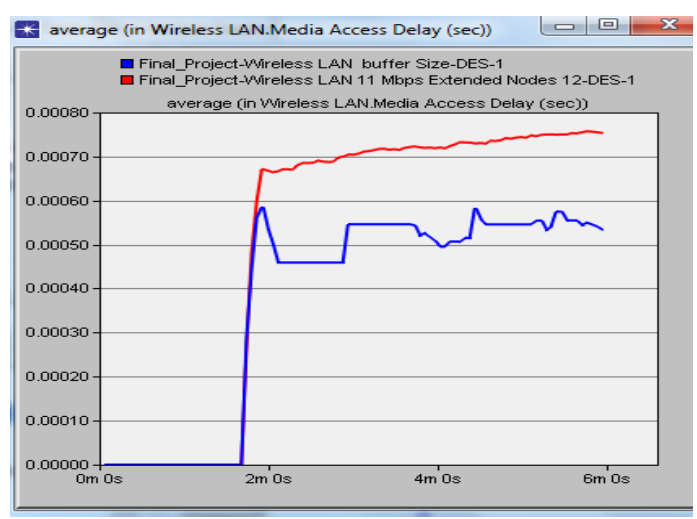

Figure 13 Media Access Delay for varying buffer size

The investigations show that the network attains the maximum throughput using IR layer. The worst results are achieved when IEEE 802.11 protocol uses FHSS layer. But an important thing to focus on is, that the throughput may vary according to the type of the network modelled, the network objects variation may occur in terms of number of stations, data rate and type of network load too among certain other parameters. The effect of change in physical characteristics on throughput i.e. on the bit rate sent to the higher layer is shown in Figure 12.

Buffer size (bits) specifies the maximum size of the higher layer data buffer in bits. Once the buffer limit is reached, the data packets arrived from higher layer will be discarded until some packets are removed from the buffer, so that the buffer has some free space to store these new packets. The optimum size of buffer can stabilize the queue size, the packet drop probability and hence the packet loss rate. The benefits of stabilizing queues in a network are high resource utilization. When the queue buffer appears to be congested the packet discard probability increases. On the other hand, the buffer overflow can be used to manage congestion. The performance analysis has been done for a buffer size of 256kbits and 1024kbits.

The buffer configuration defines the buffer size, the maximum allocated bandwidth and minimum guaranteed bandwidth. If an incoming flow suddenly becomes bursty, then it is possible for the entire buffer space to be filled by this single flow and other flows will not be serviced until the buffer is emptied. If the buffer size is increased, (Figure 14) then the number of retransmission attempts would be reduced. 
Also the size of the queue will be decreased for larger buffer due to the fact that the larger buffer will take less time to send the packets, so the queue size will not build up continuously for larger buffer. This shows the reduction in delay as shown in Figure 13. The result of packet loss is the change of queue length. When packet loss is relatively low, packets usually can be transmitted without retransmission, so the queue length may be relatively small. But when the packet loss is high, the MAC packet retransmission (Figure 14) will prolong the delay of packet (Figure 13). So, a small buffer size can increase packet drop rate and hence change the queue length and thus impact the throughput and delay. Hence, the performance has been improved by increasing the buffer size.

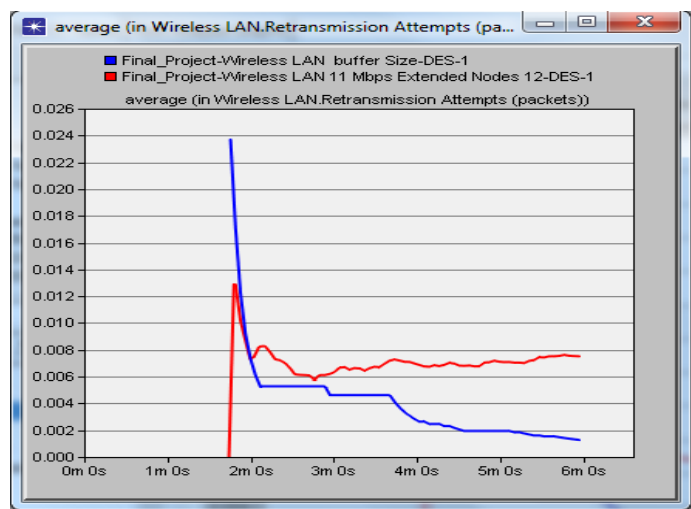

Figure 14 Retransmission Attempts for varying buffer size

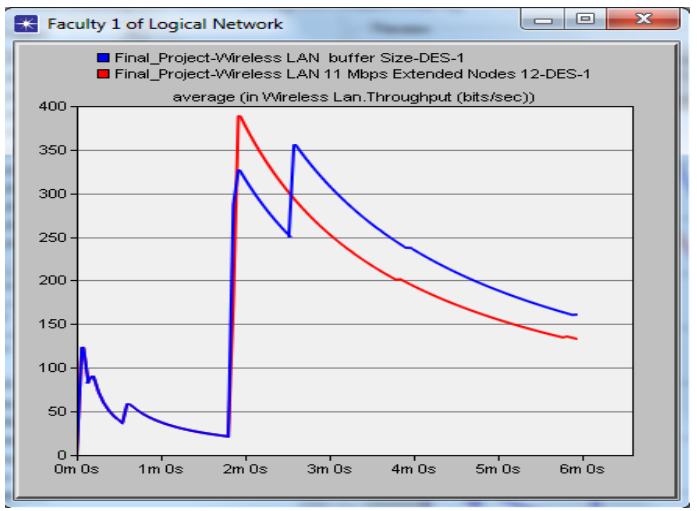

Figure 15 Throughput for varying buffer size (Node level)

The increase of packet discard rate can lead to the decrease of throughput. This happens due to frequent retransmissions of the MAC layer data packets when the packet loss rate increases. But the packet loss may happen due to low buffer size. The analysis in figure 15 shows that if buffer size is increased then the retransmission attempts would be reduced as the size of the queue is decreased for a large buffer size. The time to deliver the packets decreases, due to large buffer size. The throughput always increases monotonically with the buffer size, reaching a maximum above a threshold buffer size. Similarly, the performance analysis can be done for varying data rates, RTS threshold and fragmentation Threshold. Thus the throughput can be increased by increasing the buffer size because on increasing the buffer size, the packet drop may be reduced.

\section{CONCLUSIONS}

The impact of various network configurations on the network performance was analyzed using the network simulator- OPNET. It has been investigated that performance of the wired Networks is good if high speed Ethernet links are used under heavy network loads. The mechanism of load balancing also improves the performance by reducing and balancing the load equally among multiple servers. This lowers the response time to access server. In addition performance analysis of wireless computer networks has been done for improving the performance of wireless LAN. The investigations of physical characteristics reveal that the infrared type is best in terms of throughput. The variation in buffer size varies the queue size and hence optimizes the throughput.

\section{REFERENCES}

[1] Sarah Shaban, Dr. Hesham M.El Badawy, Prof. Dr. Attallah Hashad, "Performance Evaluation of the IEEE 802.11 Wireless LAN Standards," in the Proceedings of the World Congress on Engineering2008 , vol. I, July 2-4, 2008.

[2] Sameh H. Ghwanmeh, "Wireless network performance optimisation using Opnet Modeler," Information Technology Journal, vol. 5, No 1, pp. 18-24, 2006.

[3] Ranjan Kaparti, "OPNET IT Guru: A tool for networking education," REGIS University.

[4] “Opnet_Modeler_Manual," available at http://www.opnet.com

[5] T.Velmurugan, Himanshu Chandra and S. Balaji, "Comparison of Queuing disciplines for Differentiated Services using OPNET," IEEE, ARTComm.2009, pp. 744-746, 2009.

[6] Yang Dondkai and Liu Wenli, "The Wireless Channel Modeling for RFID System with OPNET," in the Proceedings of the IEEE communications society sponsored $5^{\text {th }}$ International Conference on Wireless communications, networking and mobile computing, Beijing, China, pp. 3803-3805, September 2009.

[7] Ikram Ud Din, Saeed Mahooz and Muhammad Adnan, "Performance evaluation of different Ethernet LANs connected by Switches and Hubs," European Journal of Scientific Research, vol. 37, No 3, pp. 461-470, 2009.

[8] J Schreiber, M K Joopari and M.A. Rashid, "Performance of video and video conferencing over ATM and Gigabit Ethernet backbone networks," Res. Lett. Inf. Math. Sci., vol. 7, pp. 19-27, 2005

[9] X Chang, "Network simulations with OPNET," Proceedings of the 1999 Winter Simulation conference, pp.307-314, 1999.

[10] Shufang Wu, Mahmood Riyadh, Riadul Mannan, and Ljiljana Trajkovic, "OPNET Implementation of the Megaco/H.248 protocol: multi-call and multiconnection scenarios," OPNETWORK 2004 Washington, DC, August 2004

[11] Yan Huang, Jian Huang, Lance Hester , Anthony Allen,Oleg Andric, Priscilla Chen, Bob O'Dea, "Opnet Simulation of a multi-hop self-organizing Wireless Sensor Network," in the Proceedings of OPNETWORK 2002 conference, Washington D.C, August 2002.

[12] Gilberto Flores Lucio, Macros Paredes-Farrera, Emmanuel Jammeh, Martin Fleury, Martin J. Reed, "OPNET Modeler and NS-2 : Comparing the accuracy of Network Simulators for packet level Analysis using 
a Network Test bed," WSEAS Transactions on Computers, pp. 700-707, 2- 3, July 2003.

[13] J. Theunis, B.Van den Broeck, P. Leys, J.Potermans, E. Van Lil, A. Van De Capelle, "OPNET in Advanced Networking Education," in the Proceedings to the International Conference on Networking ICN'01, Colmar France, 2001

[14] H.L. Velayos, "Automatic Wireless Networking," Doctoral thesis, Laboratory for Communication Networks, Department of Signals, Sensors and Systems, KTH Royal Institute of Technology, Stockholm, Sweden, May 2005.

[15] David Kotz and Kobby Essien, "Analysis of campuswide wireless network," in the Proceedings of eighth annual International conference on mobile computing and networking, pp. 107-118, September 2002.

[16] Mohd Nazri Ismail and Abdullah Mohd Zin, "Emulation network analyzer development for campus environment and comparison between OPNET Application and Hardware Network Analyzer", European Journal of Scientific Research, ISSN 1450216X, vol.24, No.2, pp.270-291, 2008.

[17] Dahai Zhang and Yanqui Bi, "Communication Network of Wide Area Protection System using OPNET Simulator," IEEE International Symposium on Industrial Electronics (ISIE 2009), pp. 1298-1303, July 5-8, 2009.

[18] Walid Hneiti and Naim Ajlouni "Performance Enhancement of Wireless Local Area Networks," in the Proceedings of IEEE ICTTA'06, $2^{\text {nd }}$ International Conference on Information \& Communication Technologies: from Theory to Applications, Damascus, Syria, vol. 2, pp. 2400-2404, April 2006.

[19] K Chandrashekhar and Paul Janes, "Optimal design of Wireless local Area Networks (WLANs) using simulation," Military Communications Conference, 2009. MILCOM 2009, IEEE, Boston, MA, 18-21, October 2009.
[20] Hetal Jasani and Naseer Alaraje, "Evaluating the performance of IEEE 802.11 Network using RTS/CTS Mechanism", in the Proceedings of IEEE EIT 2007, Chicago, IL, pp. 616-621, 17-20 May 2007.

[21] Puneet Rathod, Srinath Perur and Raghuraman Rangarajan, "Bridging the gap between the reality and simulations: An Ethernet case Study," IEEE 9 $9^{\text {th }}$ International Conference on Information Technology (ICIT'06), 2006

[22] Jonathan D. Gibbs and Hessam S. Sarjoughian, "Assessing the impact of a modelling tool and its support for verification and validation," in the Proceedings of the $12^{\text {th }}$ international conference on Symposium on Performance Evaluation of Computer \& Telecommunication Systems, Istanbul, Turkey,(SPECTS 2009), pp.73-80,13-16, July 2009.

[23] Petr Jurcik, Anis Koubaa, Mario Alves, Eduardo Tovar and Zdenek Hanzalek, "A simulation model for the IEEE 802.15.4 Protocol: Delay/Throughput Evaluation of the GTS Mechanism," in the Proceedings of $15^{\text {th }}$ IEEE International Symposium on Modeling, Analysis, and Simulation of Computer and Telecommunication Systems (MASCOTS07), Istanbul (Turkey), pp. 109-116, October 2007.

[24] Zhi Ren, Yong Huang, Qianbin Chen and Hongbin Li, "Modelling and Simulation of fading, Pathloss and shadowing in wireless networks," in the Proceedings of ICCTA 2009, pp. 335-343, 2009

[25] Jim M. Ng, Edward Chan and S.K. Lam, "A modular approach in the simulation of High Speed Networks," in the Proceedings of the 26th South-eastern Symposium on System Theory, 1994, Athens, OH, pp. 354-358, 20-22 March 1994. 